\title{
REVUE
}

\section{LE ROLE DU LAIT DANS LA TRANSMISSION DES BRUCELLOSES}

\author{
par \\ A. HOUDINIÈE \\ Docteur-Vétérinaire sanitaire du département de la Seine
}

En 1932, Ch. Nrcolle, devant le premier Congrès international d'Hygiène méditerranéenne a exprimé cette opinion :

" Ce que nous avons fait dans la voie de la connaissance des brucelloses est extrêmement intéressant et nous a beaucoup instruits ; mais il est actuel. lement temps de passer à d'autres exercices ; nous perdons notre temps ; des gens fort intelligents perdent leur temps. »

A l'époque en effet, la notion nouvelle de brucellose captivait nombre d'esprits, de corte que d'autres questions, aussi ou ou plus importantes, risquaiento de passer au second plan de l'actualité.

De plus on aurait pu croire, tout au moins pour le monde scientifique, épris de nouveauté, que désormais l'existence des brucelloses était connue de tous les praticiens de la médecine, que leur diagnostic serait couramment posé et qu'il aurait suffi aux bactériologistes de dresser des statistiques permettant de suivre avec attention l'épidémiologie de ces affections.

Ainsi exprimée par un savant français, cette opinion qui a pu paraître un peu brutale, a ralenti le zèle des vulgarisateurs et fait passer la question au second plan des préoccupations du jour tandis que les spécialistes de la brucellose, sans rompre leurs faisceaux continuaient à travailler le sujet. Il semble bien en effet que celui-ei soit encore loin d'être épuisé.

D'autre part, nombre de cliniciens paraissent encore ignorer les notions qui sont à la base de la prophylaxie antibrucelliqüe si l'on en juge par la rareté des déclarations officielles obligatoires de fièvre ondulante.

La maladie, comme on l'a dit, reste encore en dehors des éventualités diagnostiques envisagées par le médecin de campagne et est soủvent une maladie à erreurs de diagnostic, additionnées pour ne pas dire multipliées.

Dans ces conditions, il n'est pas étonnant que la Société de Pathologie comparée ait remis la question à l'ordre du jour en 1944 et entendu lors de sa. séance solennelle le rapport documenté de M. P. Rosst, Directeur des Services vétérinaires de Saône-et-Loire, qui en a fait la mise au point (1).

Or, tant que des moyens médicaux efficaces et pratiques ne pourront être opposés à la fièvre ondulante, les mesures de prophylaxie générale basée sur la connaissance de son épidémiologie resteront les seuls moyens de lutte.

C'est à pénétrer plus intimement cette épidémiologie que nous allons nous efforeer ici, à la lumière des renseignements rapportés par notre éminent Collègue, en insistant toutefois d'unê façon particulière sur le rôle du lait dans la transmission de la brucellose.

(1) P. Rossi. Les brucelloses. Bactériologie et épidémiologie. Revue de Pathologie comparée, $44^{\mathrm{e}}$ année, no 547 et 548 , mars et avril 1944, p. 80. 
Sans revenir sur la bactériologie des Brucella, rappelons que leur trois types microbiens. "Melitensis ", "Abortus 》, "Suis " (les deux premiers seuls existant en France) pathogènes pour l'homme, le sont aussi pour la plupart de nos animaux domestiques (cobaye, lapin, poule, chien, porc, cheval, vache, brebis, chèvre, etc.).

Chacun d'eux possède néanmoins une adaptation plus ou mòns élective qui lui confère un pouvoir pathogène plus marqué pour une espèce animale déterminée. Mais ceci ne lui retire pas la possibilité d'attaquer les autres espèces ou d'être, par elles, hébergé.

Si B. Melitensis a une préférence pour la chèvre et la brebis elle vit aussi chez la vache constituant ainsi une menace plus marquée pour l'homme.

B. Abortus, dont les bovidés sont le terrain d'élection, peut être l'agent du mal de garrot des équidés qui, puisant leur infection chez la vache, peuvent aussi la lui recéler.

Un cadeau réciproque analogue s'observe en Hongrie ou Abortus est transmis des bovidés aux pores et des pores aux bovidés.

Chez l'homme, Mélitensis vient en tête par son affinité, suivi de Suis puis de Bovis.

Ce chassé-croisé entre les diverses espèces animales ne permet pas de préjuger du germe lorsque l'on connaît celle qui a contaminé l'homme. Cependant, pour la fièvre ondulante due à Abortus, le rôle des bovins est presque exclusif.

Les Brucella, notamment Abortus, sont éminemment plastiques. Elles montrent suivant les souches de grosses variations de leurs aptitudes pathogènes. Ceci explique :

10 La rareté relative de la fièvre abortive de l'homme opposée au caractère épizootique de la maladie de Bang chez les bovidés;

$2^{\circ}$ L'évolution des caractères cliniques de cette même fièvre qui il y a dix ans se présentait comme une maladie fébrile à syadrome sudoro-algique et tend à devenir aujourd'hui une maladie viscérale;

$3^{\circ}$ Le caractère souvent sporadique de la fièvre abortive, mais aussi parfois son caractère endémique ;

$4^{\circ}$ Son évolution fréquemment bénigne opposée à ses quelques formes graves ou mortelles ;

$5^{\circ}$ L'accroissement parallèle du pouvoir pathogène de $B$. Abortus qui réussissant à vaincre la résistance de l'homme devient aussi pathogène pour le cheval.

\section{ZONE GÉOGRAPHIQUE D'INFLUENCE DES BRUCELLA EN FRANCE}

\section{A. Brucelloses animales}

Melitensis touche d'une façon massive les ovins et caprins 
du Sud-Est et du Sud-Ouest. Il atteint par épisodes, ceux de Meurthe-et-Moselle, des Vosges, de la Seine-et-Marne et d'Eure-etLoir. De plus il ne ménage pas les bovins du Sud-Est et de l'Est (Isère, Rhône, Ain, Drôme, Meurthe-et-Moselle, Haute-Savoie, Haute-Saône, Yonne).

Abortus existe partout où l'on pratique l'élevage bovin ; il est l'agent exclusif des brucelloses équines; on ne le rencontre qu'exeptionnellement chez la chèvre et la brebis ; il a provoqué quelques avortements chez les truies en Haute-Savoie, en Corrèze, dans le Pas-de-Calais et en Eure-et-Loir.

Dans l'ensemble les avortements contagieux sont en progession chez les animaux. Pour la seule espèce bovine, le pourcentage des troupeaux français infectés ne doit pas être inférieur à $15 \%$.

\section{B. Brucelloses humaines}

Depuis l'époque où les brucelloses ont été recherchées d'uné façon plus systématique, le nombre des départements infectés a été en se multipliant.

Il ne resterait d'indemne (?) que la Charente, les Côtes-du-Nord et peut-être les Deux-Sèvres.

L'enquête menée fait ressortir que de 1938 à 1943, 2.569 déclarations de fièvre ondulante ont été officiellement enregistrées.

Mais il faut grossir ce chiffre en raison de la carence du corps médical dans l'accomplissement de la formalité administrative de la déclaration. Rossi estime que 4.000 malades sont soignés annuellement pour fièvre ondulante.

Sur ces 4.000 cas, Melitensis se taille la part du lion puisque sur les souches isolées de l'homme 94,5\% furent classées Melitensis et $5,6 \%$ se sont comportées en Abortus.

Melitensis a été identifié dans 38 départements dont 13 situés au Nord du parallèle de Lyon considéré naguère comme divisant la France en une zone Sud mélitococcique et une zone Nord abortive (Aube, Charente-Inférieure, Côte-d'Or, Loir-et-Cher, Marne, Meuse, Meurthe-et-Moselle, Nord, Haute-Saône, Saône-et-Loire, Seine, Vosges, Yonne).

Par contre Abortus a été isolé dans 34 départements dont 9 au-dessus de ce même parallèle.

Le danger se révèle done de plus en plus alarmant de voir peu à peu notre pays envahi par B. Melitensis, si pathogène pour l'homme.

\section{Matières virulentes}

Les Brucella persistent plus ou moins longtemps dans l'organisme animal : 
Peu de brebis guéries conservent Melitensis. Le microbe, plus tenace chez la chèvre est éliminé de son organisme au maximum en 8 ou 10 mois.

Chez les jeunes bovidés, notamment les génisses, les Brucella sont expulsées spontanément avant accouplement. Par contre, et c'est là un fait important, la vache adulte accuse une pérennité absolue de l'infection qui se prolonge toute la vie durant. L'infection sanguine se retrouve encore chez $5,9 \%$ des vaches 6 ans après le début de l'infection. .

Mises à part les diverses voies d'élimination des Brucella, dont la plus importante est l'utérus, étudions celle du lait :

\section{a) Laits individuels.}

Toute femelle contaminée élimine, par voie mammaire, même en l'absence du moindre symptôme clinique, la Brucella.

Melitensis, mis en évidence, dès 1904, dans le lait de chèvre, fut retrouvé chez la brebis, chez la vache et chez la femme.

$B r$. Abortus a été isolé du lait de vache, de brebis et de chèvre.

$B r$. Suis a été maintes fois rencontré en Amérique dans le lait de la vache.

Si le lait peut contenir les ' 3 types de Brucella, il n'accuse cependant aucun changement de ses caractères, de sa composition chimique et de sa cytologie. Toutefois chez la chèvre, il existerait une augmentation, parfois considérable de la matière azotée corrélative d'un leucocytose plus ou moins marquée.

En raison même du caractère évolutif de l'infection, les conditions de l'excrétion bacillaire varient selón les sujets. Continue chez les autres, elle se fait tantôt par le pis tout entier, tantôt par un seul ou par plusieurs quartiers, avec des alternances mal connues.

Les différences observéès sont surtout d'ordre quantitatif. L'excrétion commence généralement avec le début de la lactation ou peu après. Massive à ce moment de pleine activité mammaire, elle atteindrait 200.000 germes par centimètre cube pour se réduire nettement pendant la période d'infection latente, où, chez la vache, 2.000 Abortus ne seraient pas dépassés.

Chez la chèvre au contraire, l'excrétion se maintiendrait élevée $(80.000,100.000$ et même 200.000 Melitensis $)$. Ceci explique en partie, la nocivité moindre de l'ingestion du lait de vache et son rôle plus discret.

Aussi abondante après les accouchement's normaux qu'après les avortements, l'excrétion subit de grandes oscillations chez un même individu, passant en 20 jours de quelques unités à 4.000 puis 10.000 germes au centimètre cube. 
Sa persistance dépend de l'espèce et de l'individu : arrêtée chez la chèvre et la brebis, entre le $20^{\circ}$ et le $60^{\circ}$ jour, elle peut être observée après ce dèlai, même pendant 140 jours.

Par contre chez la vache, l'excrétion peut persister toute la vie. Chez cette femelle, on a indentifié Melitensis après 18 mois et Abortus après trois ans. Le record semble revenir à la vache de Schroeder et Cotton, toujours bacillifère après 7 ans.

La recherche des agglutinines dans le sang et le lait permet-elle de conclure à l'existence des germes dans le lait ?

La majorité des auteurs admet qu'une séro-agglutination au dessous de $1 / 50^{e}$ traduit une intégrité du lait qui serait, au contraire infecté lors d'agglutination à $1 / 200^{\mathrm{e}}$ et au-dessus.

D'après Lerche le nombre de vaches excrétant des Brucella augmente avec le titre de l'agglutination.

Cependant la littérature contient un nombre impressionnant de travaux relatant que $18 \%$ des porteurs de germes $(3,5 \%$ seulement d'après RINJARD), fournissant un lait à Brucella, peuvent avoir une épreuve sérologique négative, soit que le sérum ne contienne aucune agglutinine, soit que la quantité de celles-ci soit insuffisante pour indiquer nettement l'infection.

D'autre part, il n'existe guère de rapport entre le pouvoir agglutinant du lacto-sérum et l'excrétion de Brucella par la mamelle.

Toute lacto-agglutination à $1 / 20^{\mathrm{e}}$ soulignerait la présence du bacille. En pratique, cette méthode de laboratoire ne dépiste que $80 \%$ des cas et peut être négative bien que le lait renferme des microbes.

Pour certains, le fait que le pouvoir agglutinant du lactosérum l'emporte sur celui du sérum sanguin est un signe de la multiplication des Brucella dans le tissu mammaire.

En général, les deux tiers des vaches infectées, donnant un séro-diagnostic positif ont aussi un lacto-diagnostic positif.

Chez la plupart-de nos grands animaux, l'apparition des bacilles précéderait, et souvent de beaucoup ( 8 mois) celle des séro et lacto-agglutinines.

\section{b) Laits du commerce et produits laitiers.}

Le mélange des laits entraîne une large dilution microbienne. Les Brucella restent cependant vivantes pendant toute la période d'utilisation du lait, conservent leur vitalité et ne diminuent pas en nombre.

On manque de statistiques françaises sur le degré de pollution des laits de mélange offerts à la consommation ou servant à la préparation de produits dérivés. 
A Paris, sur 26 laits crus, Verge n'en a trouvé qu'un de suspect. Le pourcentage des contaminations est évalué à 24,8 à Varsovie et 51,5 à Breslau.

Les laits pasteurisés n'offrent pas toutes garanties parce que si théoriquement le chauffage tue les Brucella, la pasteurisation est souvent mal exécutée.

La contamination du lait entraîne celle de tous ses dérivés. En beurrerie, le taux des Brucella dans le beurre ou ses sous-produits est variable avec les techniques utilisées. Très élevé dans la crème d'écrémage spontané et par suite très bas dans le lait écrémé restant, il est par contre peu élevé dans la crème de centrifugeuse.

Le fouettage de la crème ne diminue pas cette richesse ; gâteaux à la crème et glaces sont donc une source d'infection à retenir dans les pays où leur consommation est importante. A Turin, sur 10 échantillons de "panna montata ", PAGNINI a trouvé 2 fois Melitensis et 23 fois Abortus.

Lors du barattage et du malaxage, la plupart des bacilles sont remis en suspension dans le babeurre. Le beurre n'en contient donc que des quantités relativement faibles qui diminuent encore si un nouveau lavage est opéré.

Cette épuration mécanique est complétée par une action chimique due à la maturation de la crème, véritable fermentation lactique. Si celle-ci n'a pas le temps d'agir sur les laits commerciaux, son rôle épurateur est capital pour les dérivés puisqu'elle détruit la Brucella en 48 heures et même plus vite dès que l'acidité arrive à $40^{\circ} \mathrm{D}$. Si elle est plus modérée et ne dépasse pas 25 à $30^{\circ}$, elle permettrait la survie de Melitensis pendant quarante-cinq jours dans le beurre.

Celui-ci serait alors, en principe, avirulent, bien-qu'on ait retrouvé la Brucella vivante au bout de 5 mois.

A ce sujet nous nous permettrons de signaler qu'il y aurait intérêt à être fixé sur le devenir des Brucella dans les beurres de crème douce dont la fabrication a été récemment recommandée en France.

La Brucella conserve sa vitalité et son pouvoir pathogène dans les fromages non fermentés. Les épidémies, citées par CARRIEU et La Fenetre (fromages de chèvres), celles de Veloppe et JAUBERT (brousse de brebis), celles,observées en 1942 à Tonneins par Rossi sont très démonstratives à cet égard.

Les fromages affinés au contraire n'ont jamais été accusés (Roquefort). Le Saint-Marcellin qui demande 3 mois de maturation ne recèle pas de Brucella Metitensis. 


\section{Voies de pénétration}

Chez les humains e'est la voie cutanée qui est le plus souvent empruntée par le microbe et qui constitue pour lui la voie idéale (petites écorchures, crevasses...).

Cependant la voie digestive, moins sûre, n'a pas un rôle négligeable : expérimentalement la pénétration digestive réussit dans $22 \%$ des essais, soit 4 fois moins que l'introduction par la peau.

\section{Modes de contamination}

Chez les animaux la contamination est réalisée de multiples façons (accouplement, alimentation, contact cutané, etc.). Ses modes varient d'ailleurs avec l'espèce animale. Ainsi chez les bovins, le rôle de l'accouplement est de peu d'importance. Par contre, il est plus sérieux chez les ovins et caprins en raison des répétitions rapides des saillies. Chez le pore, l'accouplement serait le facteur primordial de propagation.

En ce qui concerne le lait virulent, son ingestion, dangereuse chez les porcins adultes, n'a qu'un rôle insignifiant dans la contamination des jeunes veaux qui, tous, offrent une résistance invincible à la brucellose et peuvent être impunément alimentés avec du lait infecté.

En fait, la contamination par la peau (membres postérieurs et mamelle) paraît être la plus importante chez les animaux.

Chez l'homme, dans presque tous les cas, la fièvre ondulante a pour point de départ une infection animale. Cependant elle peut être d'origine humaine ou due à une contamination de laboratoire.

Dans le premier cas, seul envisagé ici, quel est le rôle réel du lait ?

D'une étude comparative poursuivie par le Professeur LisBONNE entre 470 sujets ayant contracté la fièvre ondulante et 2.827 ne l'ayant pas contracté dans un milieu essentiellement infecté, il ressort que sur les 825 qui consommaient du lait, 124 eurent la fièvre ondulante et 701 furent épargnés.

D'autre part, sur 466 cas de fièvré ondulante ayant fait l'objet d'une enquête individuelle très serrée, 37 , soit $7,9 \%$ paraissent imputables à la consommation de laitage contre 190 soit $48 \%$ au contact.

Dans l'ensemble 25 à $50 \%$ des personnes qui boivent du lait cru absorbent la Brucella; mais la contamination ne s'ensuit que rarement : elle réclame, en effet, avec de grandes quantités de germes, la multiplication des ingestions. De plus, certains auteurs admettent qu'il faut une diminution de la résistance de l'organisme tandis que d'autres croient que l'ingestion prolongée de laits. bacillifères fait naître une certaine immunité. 
Si le lait de brebis et de chèvre paraissent plus dangereux que le lait de vache, à cause de la richesse microbienne, il faut reconnaître que le lait ou les produits laitiers contenant Melitensis ne sont responsables que de $1 / 10^{e}$ des cas de fièvre ondulante. Certains même estiment leur intervention comme exceptionnelle : Ainsi Grenoble " approvisionnée par un effectif important de vaches infectées de $\mathrm{Br}$. Melitensis ne présente pas de cas autochtones de fièvre ondulante ").

"On croit, trop souvent encore, chez nous, que l'ingestion de lait cru ou de fromages frais est le seul mode de contamination de l'homme ; cette transmission indirecte par voie alimentaire est à la vérité infiniment plus rare que la transmission des germes de l'animal à l'homme." "

L'équation, désormais caduque, fièvre ondulante = lait ou, mieux lait de chèvre, reste cependant encore tellement ancrée dans l'esprit de nombreux médecins que l'alimentation, qui devrait être reléguée au second rang, guide, seule, leurs enquêtes épidémiologiques.

Avec B. abortus dont le pouvoir pathogène est moindre, la consommation de lait ou de fromage frais n'intervient pour ainsi dire pas en France.

A l'étranger l'origine lactée doit être plus régulièrement retenue $(87,5 \%$ des cas à Berlin, $85,5 \%$ en Ecosse, $45 \%$ aux U. S. A., $65 \%$ en Angleterre, $50 \%$ au Danermak). Le pourcentage est minime en Autriche et en Italie.

Si le lait avait un rôle prépondérant, les cas urbains égaleraient les cas ruraux ; or c'est l'inverse que l'on constate. Sur presque 2.000 cas étudiés par Rossi, $84,4 \%$ concernent les eampagnes et $15,5 \%$ les villes. Encore faut-il ajouter que cette proportion de fièvres ondulantes rurales est inférieure à la réalité puisque l'on considère à tort les observations relevées dans les petites agglomérations et dans les hôpitaux comme des cas urbains.

Ces derniers sont tous imputés à l'alimentation sans que l'on ait pris la précaution d'enquêter sur la possibilité d'un contact avec l'animal.

Le Français fait bouillir le lait de vache et réserve pour la préparation des fromages, les laits de brebis et de chèvres.

L'ébullition, tout en étant éminemment inídiquée, joue surtout vis-à-vis des populations des villes : elle ne semble pas, en maintes occasions, avoir la moindre influence sur la disparition des épidémies à la campagne.

La notion classique de la transmission alimentaire est ainsi située à sa place réelle sans pour cela être négligée tout à fait. 
En effet, dans la réalité, la contamination la plus fréquente est la contamination directe ou professionnelle.

La maladie frappe surtout les personnes qui soignent les animaux ou qui manipulent les produits infectés comme le prouvent encore les dernières statistiques établies en Sảone-et-Loire: sur 84 cas : manœuvres obstétricales, 44 ; contact sans distinction, 21 ; lait, 3 ; causes indéterminées, 16.

On en trouve une autre preuve dans le fait que la fréquence des fièvres ondulantes est en relation directe avec l'importance des troupeaux.

$$
\text { *** }
$$

La prémunition des animaux par les cultures vivantes n'est-elle pas susceptible de créer des foyers dangereux pour l'animal et l'homme?

C'est l'opinion formelle de nombreux chercheurs et en particulier de :

$1^{\circ}$ Rотн, cet auteur estime qu'en Allemagne la prémunition est la cause de la multiplication des cas de brucellose d'origine bovine : "le procédé a augmenté considérablement le nombre des vaches porteuses de germes et contamine de la sorte le lait du commerce " ;

$2^{\circ}$ Lisbonne et Merliad qui, en France, en apportent une preuve cruciale : l'unique vache d'une petite exploitation fut prémunie au moyen d'un vaccin vivant, bien que n'ayant jamais présenté le moindre signe d'avortement épizootique ; elle contamina sa propriétaire. De l'hémoculture humaine et du lait de la vache fut isolé un Abortus légèrement atypique dont la parfaite identité fut établie ultérieurement avec la souche ayant servi à la préparation du vaccin.

$$
* * *
$$

Nous avons vu que les jeunes animaux résistent à l'infection. Les tout jeunes porcelets par exemple supportent, pendant trois mois et demi à 4 mois, l'administration quotidienne "per os" de 330.000 à 2 millions d'Abortus contenus dans des laits bacillifères. Les agneaux sont également très résistants.

Il en est de même pour les nourrissons et les enfants. Les bébés nés de femmes malades et dont le lait contient Melitensis échappent à la contagion.

Cette rareté de la brucellose infantile due à une résistance innée s'explique aussi par le contact moins étroit des enfants avec les animaux.

L'infection exceptionnelle chez les moins d'un an, très rare 
de 1 an à 4 ans, rare au-dessous de 15 ans, frappe surtout les adultes, notamment ceux de 30 à 50 ans.

Les femmes sont les plus atteintes du fait de leurs occupations campagnardes.

La maladie est plus fréquente à certaines époques (agnelage, mise-bas) où la contamination estaplus intense. Là où les naissances animales sont échelonnées le long de l'année, la fièvre ondulante est moins périodique.

Si par rapport aux animaux l'homme n'est cependant frappé que d'une façon minime c'est en raison :

$1^{0}$ De la promiscuité et de la cohabitation étroite où sont entretenus les animaux dans des locaux infectés opposées aux conditions plus saines de vie des humains ;

$2^{\circ}$ De la résistance nette de l'homme brisée seulement dans certaines circonstances (surmenage, fatigue, sous-alimentation, etc.).

Ce dernier facteur joue-t-il chez les animaux?

Les uns soutiennent que l'extension de l'avortement épizootique provient d'un amenuisement de la résistance animale par l'exagération de la production laitière.

Les autres ne voient là qu'une modification de la virulence des Brucella.

$$
\text { *** }
$$

A la lumière de ces quelques documents, on peut done dire que les notions nouvelles acquises en ces dernières années sur l'épidémiologie de la brucellose viennent, quant au lait, confirmęer ce que l'on savait déjà sur son rôle dans l'étiologie de la fièvre ondulante, savoir: Le lait n'est qu'un facteur secondaire dans la transmission de la brucellose parce qu'il est beaucoup moins souvent à l'origine des cas de brucellose humaine que les autres facteurs de contamination et surtout parce que le danger qu'il représente peut-être facilement et parfaitement évité par la pasteurisation et l'ébullition domestique des laits suspects. Le problème est différent pour certains dérivés du lait (fromages frais).

Sans minimiser l'intérêt et l'importance de cette contamination d'origine lactée, il n'est plus permis à tous ceux qui s'intéressent à l'hygiène du lait ou qui doivent s'y intéresser du fait de leur responsabilité professionnelle, tels les praticiens de la médecine, d'ignorer plus longtemps cette notion fondamentale (1).

(1) Note de la Rédaction : Le Lait produira dans son prochain numéro le texte d'une récente communication de M. le Professeur Lisbonne, au sujet de l'extension de la brucellose humaine provoquée par le lait de chèvre. 